\title{
COMPARISON OF STOCHASTIC PREDICTION MODELS BASED ON VISUAL INSPECTIONS OF BRIDGE DECKS
}

\author{
Ivan ZAMBON ${ }^{\mathrm{a}}$, Anja VIDOVIC ${ }^{\mathrm{a}}$, Alfred STRAUSS ${ }^{\mathrm{a}}$, Jose MATOS ${ }^{\mathrm{b}}$, Joao AMADO ${ }^{\mathrm{c}}$ \\ a Department of Civil Engineering and Natural Hazards, University of Natural Resources and Life Sciences, \\ Peter Jordan Strasse 82, A-1190, Vienna, Austria \\ ${ }^{b}$ Civil Engineering Department, Minho University, Campus de Azurém, 4800-058 Guimarães, Portugal \\ ${ }^{c}$ Asset Management of Infraestruturas de Portugal, S.A., Estação de Sta. Apolónia, Sala 249, \\ 1100-105 Lisbon, Portugal
}

Received 06 Mar 2017; accepted 24 Apr 2017

\begin{abstract}
Due to a considerable amount of information required to support the decision-making processes, an increasing number of infrastructure owners use computerized management systems. Bridges, being complex and having significant impact on society, have often been the foundation for the development of these systems. In order to manage bridges effectively, condition prediction models are incorporated to the core of decision-making processes. Many of developed and applied stochastic prediction models show certain limitations. The impact of these limitations on deterioration predictions cannot be objectively evaluated without direct comparison of prediction results. Hence, several stochastic prediction models based on condition ratings obtained from visual inspections of bridge decks are compared in this article. Models are described and implemented on the data of around 1100 reinforced concrete bridge decks from the 'Infraestruturas de Portugal', a state owned Portuguese general concessionaire for roadways and railways. The statistical analysis of different models revealed significant deviations, particularly in higher condition ratings. Results indicate limited prediction capability of a simple homogeneous Markov chain model when compared with time- and space-continuous models, such as the gamma process model.
\end{abstract}

Keywords: stochastic prediction models, Markov process, gamma process, bridge management system, condition rating, visual inspection.

\section{Introduction}

Transport is essential for the development because it enables trade between people and plays an important part in economic growth and globalization. A transportation network is a realisation of a spatial network consisting of fixed installations (Barthelemy 2011). Being such installations, bridges are crucial segments of roadway and railway transportation networks, and require timely decision making for Maintenance, Repair and Rehabilitation (MR\&R) activities. To cope with demands of huge networks, Bridge Management Systems (BMSs) have been developing since the early 1990's to manage bridges reliably ( $\mathrm{Bu}$ et al. 2011). The fundamental objective of these systems is to help the operators establish an effective operation strategy as a compromise between technical and social factors, such as maximization of network performance, minimization of life-cycle costs, and minimization of the probability of failure (Bortot et al. 2006).

The forecast of long-term performance expressed through a deterioration model is one of the main components of BMSs. Numerous deterioration models are developed to both determine the bridge remaining usage period and to meet MR \& R needs (Karunarathna et al. 2013). Deterioration models can be divided into mathematical (statistical), empirical and physical, where among mathematical models Markovian models are most frequently used. Most of BMS software utilizes Markovian models that are based on condition ratings of bridge components or of an overall bridge in a network (Mirzaei et al. 2014). Condition ratings are obtained in visual inspections and assigned to different scales by different organizations.

There are several important issues concerning the use of a homogeneous Markov chain for management optimization. These issues can be found described in detail by Frangopol et al. (1997) and Agrawal et al. (2010) and it can be summarized as follows:

- in Markov chain the condition state is not continuous, but discrete and finite;

- transition probabilities in the transition matrix are difficult to assess non-subjectively; 
- Markov chain assumes discrete transition time intervals, constant bridge population, and stationary transition probabilities, which are sometimes deemed impractical;

- Markovian chain models currently implemented in advanced BMSs use the first-order Markovian decision process that assumes duration independence for simplicity, i.e. the future facility condition depends only on the current facility condition and not on the facility condition history, which is unrealistic;

- transition probabilities assume that the condition of a facility can either stay the same or decline.

Due to the shortcomings of Markov chain in modelling deterioration represented by condition ratings, scientific community has invested significant effort in developing new stochastic models. Part of the effort was directed at adapting and using Bayesian optimisation techniques and Artificial Neural Network (ANN) techniques, but it was mostly directed at adapting different types of Markov processes. In order to objectively assess the advantages and disadvantages of prediction models, the results of prediction need to be compared. Therefore, the main objective of this article is a comparison of several stochastic prediction models using the same database. Namely, the Markov homogeneous and inhomogeneous chain, Markov process with exponential and Weibull distribution and gamma process. In addition to comparison, this article focuses on the problematics of scarce input data, this being one of the major shortcomings in deterioration modelling, and it also indicates the possibility of using continuous-time and continuous-state space models, such as the gamma process model, as an alternative to the predominantly used Markov chain models.

\section{Bridge decks}

The bridge decks data used in this article were acquired from the "Infraestruturas de Portugal" (IP), a state owned Portuguese general concessionaire for roadways and rail- ways. The data consist of assigned condition ratings from Principal visual inspections of roadway concrete bridge decks. Visual inspections in Portugal are divided to Routine and Principal, the latter being conducted every 5 years. The condition ratings are allotted on the scale of 0 to 5, 0 being the best condition and 5 the worst (Freire, Horta 2012).

In order to generate a unique data set that is appropriate for all of the models presented in this article, several constrains are introduced. Since IP uses the philosophy of considering the condition state 4 as being critical, meaning that the structure has to be subjected to a repair action until the next inspection, this philosophy results in infrequent occurrence of condition state 5. Therefore, the condition rating 5 is affiliated to condition rating 4 , and the condition rating 4 is considered to be the upper threshold. The bridge decks older than 80 years as well as bridge decks with unknown age are discarded. In conjunction with the age constrain, the constraint of non-decreasing condition rating and the constraint of condition rating not increasing for more than one grade per inspection are introduced. Furthermore, only bridge decks with two consecutive inspections are used for calculation of probabilities of transition. Considering all identified constraints, the data set of 1127 bridge decks is generated, as shown in Table 1.

Table 1. Occurrence of events (condition ratings) at first and second inspection

\begin{tabular}{l|c|c|c|c|c|c|c}
\hline \multicolumn{1}{c|}{ Period } & CR 0 & CR 1 & CR 2 & CR 3 & CR 4 & CR 5 & $\sum$ \\
\hline $\begin{array}{l}\text { First } \\
\text { inspection }\end{array}$ & 88 & 597 & 364 & 76 & 2 & 0 & 1127 \\
$\begin{array}{l}\text { Second } \\
\text { inspection }\end{array}$ & 5 & 590 & 431 & 96 & 4 & 1 & 1127 \\
\hline
\end{tabular}

Besides condition ratings, data comprise additional information such as structural and bridge type, material and distance to the sea, as presented in Table 2. For each

Table 2. Information for bridges regarding the structure and bridge type, distance to the sea and material, with assigned number of bridges in each category

\begin{tabular}{|c|c|c|c|c|c|c|c|}
\hline Structure Type & No. & Bridge type & No. & $\begin{array}{l}\text { Distance to } \\
\text { the sea }\end{array}$ & No. & Material & No. \\
\hline Multiple span & 508 & Overpass bridge & 238 & $<2 \mathrm{~km}$ & 22 & Reinforced concrete & 759 \\
\hline Frame bridge & 202 & Bridge & 230 & $2-10 \mathrm{~km}$ & 109 & Pre-stressed concrete & 368 \\
\hline Simply supported (single span) & 174 & Underpass bridge & 226 & $10-50 \mathrm{~km}$ & 515 & & \\
\hline Box culvert & 106 & Culvert & 226 & $50-100 \mathrm{~km}$ & 249 & & \\
\hline Precast arch & 68 & Cattle creep & 115 & $>100 \mathrm{~km}$ & 232 & & \\
\hline Arch (single/ multiple span) & 38 & Viaduct & 57 & & & & \\
\hline Mixed solution (more than one type) & 12 & Footbridge & 33 & & & & \\
\hline Gerber beam & 10 & Tunnel & 1 & & & & \\
\hline Widening deck over arch & 6 & Other & 1 & & & & \\
\hline Suspension bridge & 1 & & & & & & \\
\hline Other & 1 & & & & & & \\
\hline Not known & 1 & & & & & & \\
\hline
\end{tabular}




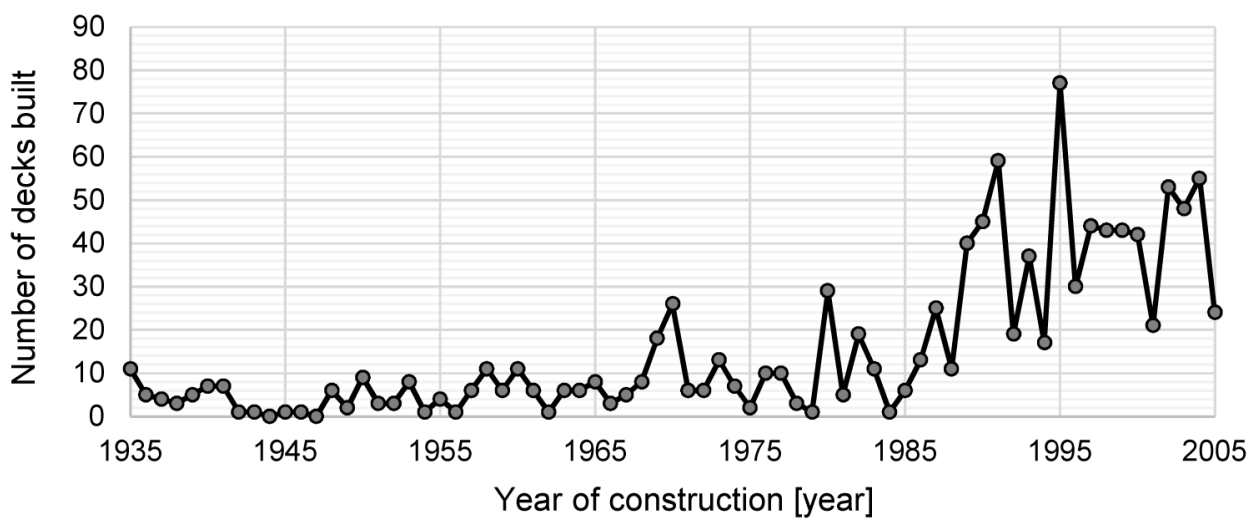

Fig. 1. Years of construction of decks used as the input for some of the models presented

category of information in Table 2, a number of bridges belonging to the category are assigned.

Consecutive inspections were performed during the period from 2005 until the end of 2015, the period between inspections being approximately 5 years long $( \pm 2$ years). In Figure 1, the years of bridge decks construction are presented. As it can be seen, most of them were built between 1990 and 2005.

\section{Markov chain}

\subsection{Introduction}

Markov processes, in general sense, describe random occurrence of changes over time by means of Markov property. This property implies that the future condition depends only on the present condition, not the sequence of events that proceeded (Feretic 2014). Markov chain is used to calculate the probability indicating the element such as a bridge deck will, at a given time, be in a particular condition state. More detailed assumptions and restrictions included in order to optimize the Markov chain model for bridge assessment can be found in works of Puz et al. (2010) and Kleiner (2001).

When the process with a number of conditions states $k$ is considered, the probability vector $p\left(t_{n}\right)$, at any given moment $t_{n}$, shows the probability that the process will assume one of the condition states $k$. This vector can be expressed by the following equations:

$$
\begin{aligned}
& p\left(t_{n}\right)=p(0) * P^{n} ; \\
& p\left(t_{n}\right)=\left\{p_{1}\left(t_{n}\right) \quad p_{2}\left(t_{n}\right) \quad p_{3}\left(t_{n}\right) \ldots p_{k}\left(t_{n}\right)\right\},
\end{aligned}
$$

where: $p\left(t_{n}\right)$ is the condition state probability vector; $p(0)$ is the initial condition state probability vector; $P$ is the transition probability matrix; $p_{i}\left(t_{n}\right)$ is the probability that the process will be in the condition state $i$ at the moment $t_{n}$.

In case of condition ratings, the initial condition state vector is formed on the basis of the latest performed deck inspections, following the Eqn (3):

$$
p(0)=\left\{p_{1}(0) \quad p_{2}(0) \ldots \quad p_{k}(0)\right\}
$$

where: $p_{i}(0)$ is the probability that the process will be in the condition state $i$ at the initial moment.

In addition to the initial condition state vector the transition probability matrix $P$ should also be known. The transition probability matrix $P$ is a square matrix of the $k^{\text {th }}$ order with elements $p_{i j}$. If during a single period of time (from $t_{n}$ to $t_{n+1}$ ) the process can either pass on to the next higher condition state or remain in the same condition state, then the transition probability matrix takes the following form:

$$
P=\left[\begin{array}{ccccc}
p_{11} & p_{12} & 0 & \ldots & 0 \\
0 & p_{22} & p_{23} & \ldots & 0 \\
0 & 0 & \ldots & \ldots & 0 \\
0 & 0 & \ldots & p_{k-1 k-1} & p_{k-1 k} \\
0 & 0 & \ldots & 0 & 1
\end{array}\right],
$$

where: $p_{i j}$ is the probability that the process will pass from the condition state $i$ to the condition state $j$ during the time period from $t_{n}$ to $t_{n+1} ; p_{i i}$ is the probability that the process will remain in the condition state $i$ during the time period from $t_{n}$ to $t_{n+1}$.

\subsection{Homogeneous Markov chain}

More commonly used Markov chain where transition probabilities $p_{i j}$ are not time-dependent is called the homogenous Markov chain. Conversely, in the inhomogeneous Markov chain the transition probabilities $p_{i j}(t)$ are time-dependent.

In order to form the transition matrix $P$, the relative frequency of occurrence of events under study needs to be determined. This is done by using the data introduced in Table 1. After determining the relative frequencies, the transition matrix $P$ takes the form:

$$
P=\left[\begin{array}{ccccc}
0.06 & 0.94 & 0 & 0 & 0 \\
0 & 0.85 & 0.15 & 0 & 0 \\
0 & 0 & 0.94 & 0.06 & 0 \\
0 & 0 & 0 & 0.96 & 0.04 \\
0 & 0 & 0 & 0 & 1
\end{array}\right] .
$$




\subsection{Inhomogeneous Markov chain}

In the Markov inhomogeneous chain, the time dependency of probability of changing the condition states is taken into consideration. Thus, the condition state probability vector from the Eqn (1) takes the form:

$$
p(t+k)=p(t) * P^{t, t+1} * P^{t+1, t+2} * \ldots * P^{t+k-1, t+k},
$$

where each time-dependent probability matrix $P^{t, t+1}$ can be expressed as:

$$
P^{t, t+1}=\left[\begin{array}{ccccc}
p_{11}^{t, t+1} & p_{12}^{t, t+1} & 0 & \ldots & 0 \\
0 & p_{22}^{t, t+1} & p_{23}^{t, t+1} & \ldots & 0 \\
\cdots & \cdots & \ldots & \ldots & \ldots \\
0 & \ldots & \ldots & p_{n-1, n-1}^{t, t+1} & p_{n-1, n}^{t, t+1} \\
0 & 0 & \ldots & 0 & 1
\end{array}\right] .
$$

To determine the time dependent probability matrix $P^{t, t+1}$ the condition state is associated with the age of decks. The transition probabilities are calculated by grouping bridge decks from the entire data into smaller groups, depending on the age of decks. Decks are divided into eight groups, with a 10 -year period between the groups, as presented in Table 3. According to Table 3 each individual age dependent probability matrix $P^{t, t+1}$ is made.

\section{Markov processes}

\subsection{Homogeneous Markov process with exponential distribution}

A continuous-time Markov process is a type of stochastic processes which passes from one state to another forming a Markov chain. The process is in a particular state for a random length of time distributed depending on the state (Ng, Moses 1998). For more detailed description of characteristics of Markov process with exponential distribution see Puz et al. $(2010,2013)$. A basic equation of the Markov process where $k$ possible conditions exist is:

$$
\begin{aligned}
p_{j}(t)= & p_{1}(0) * p_{1 j}(t)+p_{2}(0) * p_{2 j}(t)+\ldots+ \\
& p_{k}(0) * p_{k j}(t),
\end{aligned}
$$

where: $p_{j}(t)$ is the probability that process is in stage $j$ in time $t ; p_{i}(0)$ is the probability that process begins $(t=0)$ from stage $i ; p_{i j}(t)$ is the probability of transition from $i$ to $j$ in time $t$.

Transition probabilities $p_{i j}(t)$ are derived from the durations during which bridge elements remain in a given condition state called "sojourn times" (also called holding times or waiting times). If sojourn times are considered as being exponentially distributed, the transition probabilities can be solved using the properties of exponential random variables (Feretic 2014). These properties are described in Eqns (9) and (10), where $\alpha$ represents the duration of the first condition rating (first sojourn time). The same principle is applied to durations of higher condition ratings.

Let $\alpha$ be a real number and $\alpha>0$. If the random variable $W$ has probability density function (PDF):

$$
f(t)=\left\{\begin{array}{c}
0 \quad \text { for } t<0 \\
\frac{1}{\alpha} * e^{-\frac{t}{\alpha}} \quad \text { for } t \geq 0
\end{array},\right.
$$

then $W$ has the exponential distribution with parameter $\alpha$. Written as $W \sim \operatorname{Exp}(\alpha)$.

Let $W \sim \operatorname{Exp}(\alpha)$. Then for $t \geq 0$ :

$$
P(W \geq t)=e^{-\frac{t}{\alpha}}
$$

One of the possible ways of deriving the information of sojourn times is by using the principle presented in Puz et al. (2013). The other solution, the one that is used in this article, is to calculate sojourn times by taking into account the probabilities shown in Eqn (5). Where the element is considering staying in certain state until the probability of transition increases above 0.5 . Calculated sojourn times are presented in Table 4.

Table 4. Sojourn times of condition states for the exponential Markov process model

\begin{tabular}{lccccc}
\hline Condition rating & CR 0 & CR 1 & CR 2 & CR 3 & CR 4 \\
\hline Sojourn time $[y]$ & 5 & 20 & 45 & 65 & $\infty$ \\
\hline
\end{tabular}

Table 3. Transition probabilities $p_{i j}$ dependent on the age of a deck, derived for the inhomogeneous Markov chain

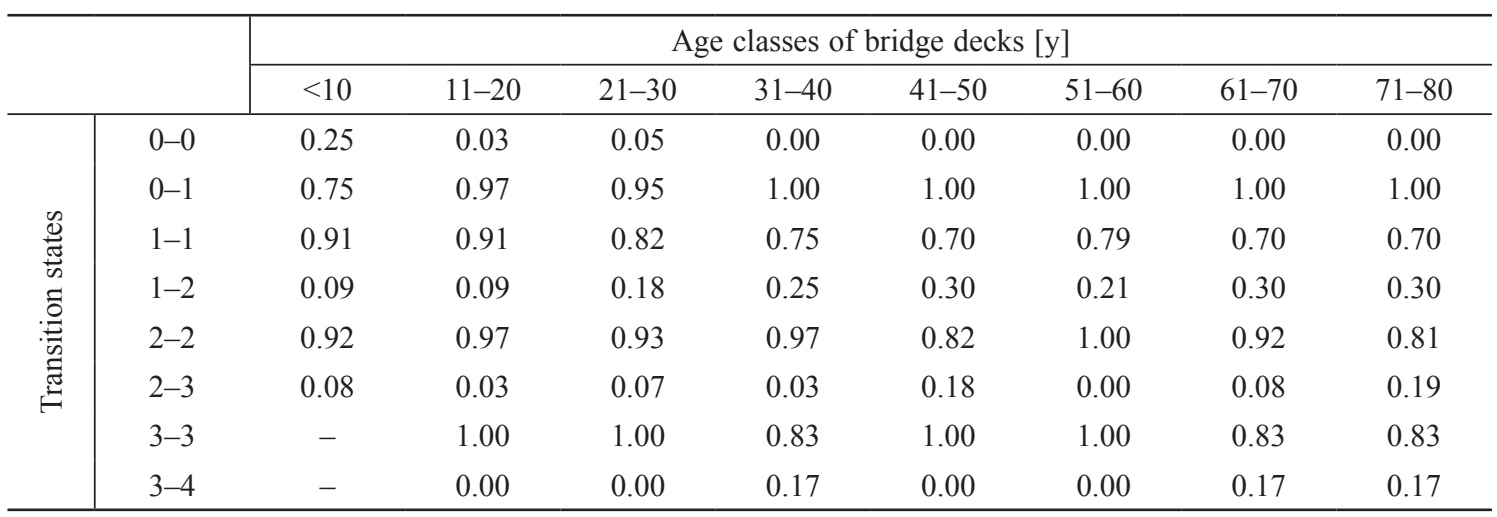




\subsection{Markov process with Weibull distribution}

In the Markov process with Weibull distribution, as well as in the previously presented Markov process based on exponential distribution, duration in each state is assumed to be a random variable with known probability distributions. The difference is that the distribution assigned to these variables is Weibull distribution, the process is not stationary, and the procedure of deriving the parameters of distribution differs. For comprehensive explanations of mathematical equations of the Markov process with Weibull distribution see Kleiner (2001).

Here, as well as in the inhomogeneous Markov chain, the condition state probability vector can be expressed through Eqn (6), and the transition probability matrix through Eqn (7). The probabilities that the process will pass from one state to the next subsequent one are calculated based on Eqn (11) and used to populate the transition probability matrix. If the process is in the state $i$ at time $t$, the conditional probability that it will transit to the next state in the next time step is expressed by:

$$
p_{i, i+1}(t)=\frac{f_{0 \rightarrow i}(t)}{S_{0 \rightarrow i}(t)-S_{0 \rightarrow(i-1)}(t)},
$$

where: $f_{0 \rightarrow i}(t)$ is the cumulative density function (CDF) of the sum of sojourn times; $S_{0 \rightarrow i}(t)$ is the survival function (SF) of the sum of sojourn times.

The CDF and SF of particular sojourn times are expressed as:

$$
\begin{gathered}
S_{i}(t)=1-F_{i}(t)=e^{-\left(\lambda_{i} t\right)^{\beta_{i}}} \\
f_{i}(t)=\frac{\partial F_{i}(t)}{\partial t}=\lambda_{i} \beta_{i}\left(\lambda_{i} t\right)^{\beta_{i}} e^{-\left(\lambda_{i} t\right)^{\beta_{i}}} .
\end{gathered}
$$

To derive parameters $\lambda_{i}$ and $\beta_{i}$ based on the historical observations and visual condition assessments of bridge decks at least two information sets in different times are to be known. The information should contain the probability (parameters $x_{i, u}$ and $x_{i, v}$ ) of the decks staying in the certain condition state for the certain duration of time (parameters $u$ and $v$ ). In the case of the data set from IP, the data from expert judgement were replaced by the data derived from the relative frequency of events, calculated similarly as for the Markov process with exponential distribution. For detailed description of procedure of deriving the parameters presented in Table 5, see Kleiner (2001).
The sum of the sojourn times is denoted as follows:

$$
t_{i \rightarrow k}=\sum_{j=1}^{k-1} t_{j, j+1}
$$

where: $i=\{1,2, \ldots, n-1\} ; k=\{2,3, \ldots, n\}$.

The $f_{i \rightarrow k}\left(t_{i \rightarrow k}\right)$ and the $S_{i \rightarrow k}\left(t_{i \rightarrow k}\right)$ of the sum of the sojourn times cannot in general be calculated analytically, therefore Monte-Carlo simulations are used to calculate these functions numerically.

\section{Gamma process model}

Unlike previous presented models, the gamma process model is both continuous-time and continuous-space model, and it has not yet been used for the prediction of condition states based on visual inspections. The gamma process is a process with independent and gamma distributed increments and it implies that deterioration progresses with frequent occurrences of small increments (AbdelHameed 1975). Comprehensive mathematical aspects of gamma process can be found in Dufresne et al. (1991) and Ferguson and Klass (1972).

Although represented by a discrete-space condition rating, physical processes causing structural deterioration (without maintenance actions) follow a non-negative and continuous function. Likewise, they are not independent of previous time-steps and consequently cannot be realistically described by the duration-independent stochastic processes. Therefore, the gamma process as a non-negative continuous function can serve as a very simple and effective method for establishing consistent deterioration models for structures subjected to inspections (Abdel-Hameed 1975). Deterioration affecting bridge decks can be wear, fatigue, corrosion, crack growth, creep, swelling, etc. If the condition ratings from visual inspections can be matched with the corresponding deterioration phenomenon, the gamma process can be easily modelled for each specific phenomenon. In that case, due to different rates of deterioration under various conditions, data would have to be partitioned into groups containing relatively consistent characteristics.

A random variable $\mathrm{X}$ that follows gamma distribution can be described using the shape parameter $a>0$, the rate parameter $\beta>0$ and the following probability density function (PDF):

$$
G a(x \mid \alpha, \beta)=\frac{\beta^{\alpha}}{\Gamma(\alpha)} x^{\alpha-1} \exp (-\beta \cdot x) .
$$

Table 5. Input parameters for the Markov process with Weibull distribution

\begin{tabular}{cccccccc}
\hline State $[\mathrm{CR}]$ & $u$ [years] & $x_{i, u}[\%]$ & $v$ [years] & $x_{i, v}[\%]$ & $\beta_{i}$ & $1 / \lambda_{i}$ & $\lambda_{i}$ \\
\hline 0 & 30 & $3.36 \mathrm{E}-08$ & 50 & $3.51 \mathrm{E}-13$ & 1 & 1.743 & 0.574 \\
1 & 30 & 0.375 & 50 & 0.195 & 1 & 30.599 & 0.0327 \\
2 & 30 & 0.676 & 50 & 0.521 & 1 & 76.603 & 0.0131 \\
3 & 30 & 0.785 & 50 & 0.668 & 1 & 124.150 & 0.0081 \\
\hline
\end{tabular}


When modelling deterioration in terms of a gamma process, the question that arises is how expected deterioration increases over time. Empirical studies show that the expected deterioration at time $t$ is often proportional to a power law (Ohadi, Micic 2011):

$$
\alpha(t)=c^{*} t^{b} .
$$

van Noortwijk and Pandey (2004) recommended using a constant exponent $b$ which can be adapted according to the considered deterioration process. In the case of deterioration represented by condition ratings, the parameter $b=0.22$ is obtained by fitting the power law formulation to the trajectory of sojourn times given in Table 4. The parameters $c$ and $\beta$ are unknown and need to be adapted through expert knowledge or statistical analyses such as the Maximum Likelihood Method, the Method of Moments or Bayesian Statistics. For that reason, the inspection periods on the transformed time axis are defined as:

$$
w_{i}=t_{i}^{b}-t_{i-1}^{b},
$$

and the deterioration increments on the transformed time axis are defined as:

$$
\gamma_{i}=\mathrm{X}_{i}-\mathrm{X}_{i-1}
$$

If the deterioration increment $\gamma_{i}$ corresponds to a gamma distribution with a shape factor $c \cdot w_{i}$ and a rate parameter $\beta$ for $i=1,2, \ldots, n$, then the population parameters can be obtained through one of the aforementioned statistical methods. Based on these assumptions, van Noortwijk (2009) recommends the following formulations for the form parameters:

$$
\frac{\hat{\mathrm{c}}}{\hat{\beta}}=\frac{\sum_{i=1}^{n} \gamma_{i}}{\sum_{i=1}^{n} w_{i}}=\frac{x_{n}}{t_{n}^{b}}=\bar{\gamma},
$$

and

$$
\frac{x_{n}}{\hat{\beta}}\left(1-\frac{\sum_{i=1}^{n} w_{i}^{2}}{\left[\sum_{i=1}^{n} w_{i}\right]^{2}}\right)=\sum_{i=1}^{n}\left(\gamma_{i}-\bar{\gamma} \cdot w_{i}\right)^{2} \text {. }
$$

The results obtained from the gamma process computation are continuous-space results, and not countable and finite-space results. Thus, in order to compare the results with the previously presented models, the finite space results made of discrete condition ratings are obtained by introducing the boundaries in continuous-space results at $0.5,1.5,2.5$, and 3.5 .

\section{Advantages and drawbacks of presented models}

Among the limitations of Markov chain model described in the introduction, the most significant effect on deterioration rates is thought to be within the stationarity of transition probabilities and duration independence. Markov inhomogeneous chain and Markov Weibull processes presented the possibility of overcoming the stationarity of transition probabilities, but they fail to take into account the dependence of duration. Conversely, the Markov exponential process does not include the age of the deck, just the probability of staying in a certain state for a certain sojourn time. Since most of the bridge decks were built between 1990 and 2005, the data are unfavourable to models with time dependent transition probabilities, especially when determining the transition probabilities of older decks. Although the input is significant when considering an overall number of decks, it is still marked with a low number of decks with high condition ratings, especially in some of the age classes. Therefore, in the case of the Markov inhomogeneous chain, the data did not present the possibility to model the time dependent transition probabilities enough convincingly, which can be seen by observing the Table 3 . Both the exponential and Weibull Markov process use the sojourn times that have to be based on expert judgement or statistically derived from input data. Theoretically, sojourn times can be improved by comparing the statistical model with physical models, i.e. with one of the models used for service life calculation according to fib Bulletin 34 (Schiessl 2006) and Bulletin 76 (Gehlen 2015). Although estimates of the time frame during which an element will stay in a certain condition resemble more to the engineering way of thinking than the concept of transition probabilities, these estimates are stil highly subjective and their influence on the outcome of prediction is crucial. Another drawback of Markov process models is that they do not take into account the length of the structural element already being in current condition state, and presume it just entered this state. This characteristic alone can cause the shift in prediction results for the whole length of the sojourn time. According to Morcous (2006), when using the Markov chain model variations in the inspection periods can result in a $22 \%$ error in predicting the service life of a bridge deck system, together with $5 \%$ of error caused by 'memoryless' assumption. Since variations in inspection periods are frequent, continuous-time models have a significant advantage compared to discrete-time models. The last presented model, i.e. the gamma process model, is a time- and space-continuous model, and it is both a nonstationary and a duration dependent model. It accounts for the past events that proceeded the current condition, thus considering every bridge deck as being distinct.

The presented models are programmed in the Matlab 2016 program, and set to summarize the probabilities of all the bridge decks in two different time horizons, first in year 2030 and second in year 2040. For every particular condition state $p_{i}\left(t_{n}\right)$ the summation of probabilities of all the data is performed, as shown in Eqn (21):

$$
p_{i, \text { set }}\left(t_{n}\right)=\sum_{j=1}^{n=1127} p_{i, j}\left(t_{n}\right) \text {, }
$$

where: $p_{i, s e t}$ is the probability of every particular condition state of the whole data set; $p_{i}, j$ is the probability of every particular condition state of every bridge deck. 


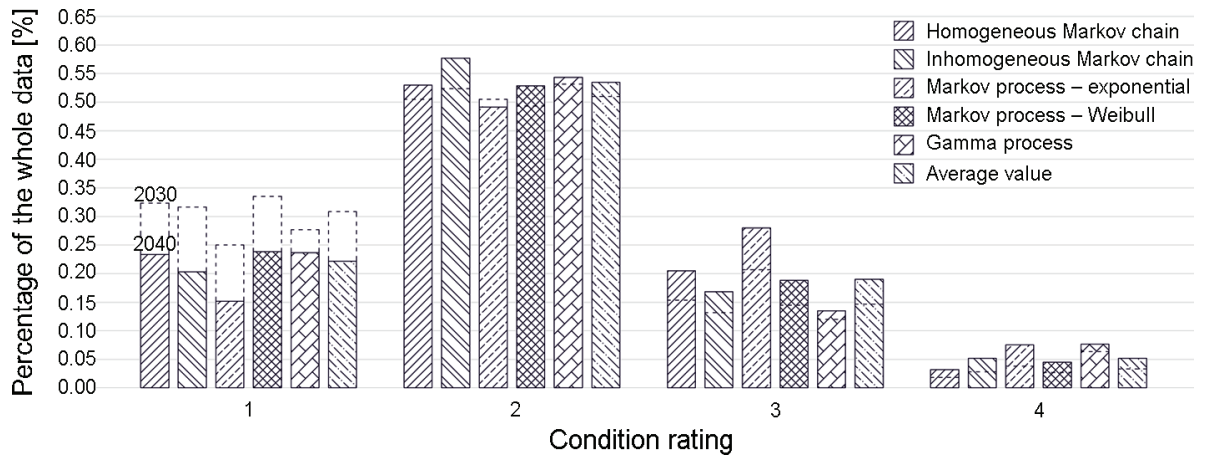

Fig. 2. Comparison of the predicted condition of bridge decks by year 2030 (striped) and 2040 , expressed as the percentage of decks being in a certain condition state; with the presumption of no interventions being conducted in the meanwhile

The calculated results are compared and presented as percentages of all decks in Figure 2, that also encompases an average value of all the models. In Figure 2, the results for 2030 are depicted with striped lines. Outlined results indicate the percentage of decks being in a certain condition state with a presumption that in the interim no maintenance or repair intervention was performed.

All of the models predicted that non of the decks will stay in the condition state 0 for both time horizons. No significant variance can be seen between the first and second time horizon in terms of relative difference between models. In other words, their comparison remains simmilar in changing the time horizons. It can be seen, from the results presented in Figure 2 that the homogeneous Markov chain predicted the lowest number of bridge decks being in condition state 4 , with only $3 \%$ of decks being in condition state 4 by 2040 . This is due to very low probability of transition from CR 3 to $\mathrm{CR} 4$, of only 0.04 (shown in Eqn (5)). Markov inhomogeneous chain predicted the highest number of bridge decks being in condition state 2 by 2040 , this being due to very high probabilities (even unrealistic for some age classes) of staying in the condition state 2 presented in Table 3. Markov exponential process in general shows the most abrupt transitions to higher condition states among all the models. Although the sojourn times in Table 4 are more than substantially lengthy, still this model predicts the highest results. The Markov process with Weibull distribution is in its prediction most alike to the simple homogeneous Markov chain. Even though the sojourn times are calculated on the same principle as for the exponential process, and both of these models are based on continuous-time processes, the results obtained differ noticeably. For the condition state 4, both the Markov exponential process and the gamma process predict almost three times the value of the homogeneous Markov chain. To some extent, regarding the gamma process, this can be explained by the fact that gamma process does not exclude the probabilities of bridge decks progressing for more than one condition rating per inspection.

\section{Conclusions}

Several stochastic prediction models based on the input data made of visual inspection condition ratings are compared in this article; additionally, an alternative continuous gamma process model was proposed. The comparison is made using the input database comprising of condition ratings gained from Principal visual inspections of reinforced concrete bridge decks provided by "Infraestruturas de Portugal". Data are characterized by a low count of consecutive inspections and a low number of bridge decks with high condition ratings, causing difficulties in calculating transition probabilities of higher condition states. Such a drawback, in case of ample information on decks locations being available, could be somewhat mitigated by sub-dividing the data into a number of groups with a similar environmental impact, in order to differentiate to a greater degree those decks with a high rate of deterioration from those deteriorating at a slower pace. Moreover, besides accounting for location and environmental impact, models should be further developed to take into account repair and preservation methods.

Shortcomings of prediction models, combined with a natural variability of deterioration, can cause high variation in results when compared with real performance of structures. To reduce possible errors arising during the estimation process, the applied model should be able take into account different deterioration phenomena in conjunction with a specific historic deterioration path of every affected element. Hence, considering all modelling advantages, the gamma process model presents a suitable alternative to the simple homogeneous Markov chain model, as well as other rendered models.

Considering the condition prediction results shown, especially for the crucial condition states CR 3 and CR 4 , high deviation in prediction can be seen with different models. Depicted results raise doubt when considering prediction accuracy of the simple homogeneous Markov chain in case of scarce input data. Since it predicts the lowest number of bridges that require intervention, irrespective of the prediction time horizon, it possibly triggers inadequate selection of maintenance/repair operation at an inadequate moment in time. 


\section{Acknowledgements}

The authors of this paper would like to acknowledge the contribution to this research made by their colleagues at the Institute of Structural Engineering at University of Natural Resources and Life Sciences in Vienna, as well as by the colleagues at the Civil Engineering Department, University of Minho in Guimarães. Likewise, the authors would like to acknowledge the Portuguese national roadway and railway manager "Infraestruturas de Portugal" for providing the support and necessary input data. The cooperation established in this article is based upon work from COST Action TU 1406, supported by COST (European Cooperation in Science and Technology). Moreover, the authors would also like to offer their gratitude for the support received through the project LeCIE "Lifecycle assessment for engineering structures in railway construction - strategies and methods", funded by ÖBBInfrastruktur Bau AG, Vienna.

\section{Disclosure statement}

The study was partially funded by the University of Natural Resources and Life Sciences Program Project LeCIE "Life-cycle assessment for engineering structures in railway construction - strategies and methods" with funds from OEBB "Austrian Railway Agency". Furthermore, this article is partially funded and it is based upon work from COST Action TU 1406, supported by COST (European Cooperation in Science and Technology). Additionally, Jose Matos works at University of Minho and Joao Amado works at "Infraestruturas de Portugal".

\section{References}

Abdel-Hameed. 1975. A gamma wear process, IEEE Transactions on Reliability 24(2): 153-153.

Agrawal, A. K.; Kawaguchi, A.; Chen, Z. 2010. Deterioration rates of typical bridge elements in New York, Journal of Bridge Engineering 15(4): 419-429. https://doi.org/10.1061/(ASCE)BE.1943-5592.0000123

Barthelemy, M. 2011. Spatial networks, Physics Reports 499(13): 1-101. https://doi.org/10.1016/j.physrep.2010.11.002

Bortot, F.; Zonta, D.; Zandonini, R. 2006. A bridge management strategy based on future reliability and semi-Markov deterioration models, in $3^{\text {rd }}$ International ASRANet Colloquium, 10-12 July 2006, Glasgow.

Bu, G.; Lee, J.; Guan, H.; Blumenstein, M.; Loo, Y.-C. 2011. Improving reliability of Markovian-based bridge deterioration model using artificial neural network, in $35^{\text {th }}$ International Symposium on Bridge and Structural Engineering (IASBE), 2011, London, United Kingdom.

Dufresne, F.; Gerber, H. U.; Shiu, E. S. 1991. Risk Theory with the gamma process, ASTIN Bulletin 21(2): 177-192. https://doi.org/10.2143/AST.21.2.2005362
Feretic, S. 2014. Markov processes and assessment of condition of bridges. University of Rijeka, Faculty of Civil Engineering, Rijeka, Croatia.

Ferguson, T. S.; Klass, M. J. 1972. A representation of independent increment processes without Gaussian components, The Annals of Mathematical Statistics 43(5): 16341643. https://doi.org/10.1214/aoms/1177692395

Frangopol, D. M.; Kallen, M. J.; van Noortwijk, J. M. 1997. Probabilistic models for life-cycle performance of deteriorating structures: review and future directions, Structural Engineering Materials 6: 197-212. https://doi.org/10.1002/pse. 180

Freire, L. R.; Horta, C. S. 2012. SGOA - Balanço de um ciclo de inspeções 2007-2011, in ICDS12-International - Conference Durable Structures: from Construction to Rehabilitation, 31 May - 1 June 2012, Lisbon, Portugal.

Gehlen, C. 2015. Benchmarking of deemed-to-satisfy provisions in standards: durability of reinforced concrete structures exposed to chlorides; fib Bulletin 76. Germany: DCC Document Competence Center Siegmar Kästl e. K.

Karunarathna, W.; Zhang, T.; Dwight, R.; El-Akruti, K. 2013. Bridge deterioration modeling by Markov Chain Monte Carlo (MCMC) simulation method. Hong Kong, s.n.

Kleiner, Y. 2001. Scheduling inspection and renewal of large infrastructure assets, Journal of Infrastructure Systems 7(4): 136-143. https://doi.org/10.1061/(ASCE)1076-0342(2001)7:4(136)

Mirzaei, Z.; Adey, B. T.; Klatter, L.; Thompson, P. D. 2014. The IABMAS bridge management committee overview of existing bridge management system. International Association for Bridge Maintenance and Safety - IABMAS.

Morcous, G. 2006. Performance prediction of bridge deck system using Markov chain, Journal of Performance of Constructed Facilities 20(2): 146-155.

https://doi.org/10.1061/(ASCE)0887-3828(2006)20:2(146)

Ng, S.-K.; Moses, F. 1998. Bridge deterioration modelling using semi-Markov theory, in N. Shiraishi, M. Shinozuka, Y. K. Wen. Structural safety and reliability, Vol. 1. Rotterdam: Balkema, 114-120.

Ohadi, A.; Micic, T. 2011. Stochastic process deterioration modelling for adaptive inspections, in M. H. Faber, J. Köhler; K. Nishijima (Eds). Applications of statistics and probability in civil engineering. Zurich: Taylor \& Francis Group. https://doi.org/10.1201/b11332-163

Puz, G.; Radic, J.; Stipanovic-Oslakovic, I. 2010. A new model for stochastic analysis of bridge durability, Gradevinar 62(4): 287-297.

Puz, G.; Radic, J.; Tenzera, D. 2013. bridge condition forecasting for maintenance optimisation, Građevinar 65(12): 1079-1088.

Schiessl, P. 2006. Model code for service life design of concrete structures, fib Bulletin 34. International Federation for Structural Concrete (fib), Lausanne.

van Noortwijk, J. M.; Pandey, M. D. 2004. A stochastic deterioration process for time-dependent reliability analysis, in Proceedings of the Eleventh IFIP WG 7.5 Working Conference on Reliability and Optimization of Structural Systems, 2-5 November 2003, London, United Kingdom. van Noortwijk, J. M. 2009. A survey of the application of gamma processes in maintenance, Reliability Engineering and Safety 94: 2-21. https://doi.org/10.1016/j.ress.2007.03.019 
Ivan ZAMBON. Ivan Zambon is a PhD student in the Department of Civil Engineering and Natural Hazards at University of Natural Resources and Life Sciences, Vienna. He received his MS from Faculty of Civil Engineering at University of Rijeka in 2014. His research interests are assessment and evaluation of existing structures with the emphasis on material durability and deterioration mechanisms.

Anja VIDOVIC. Ms Vidovic received her MSc degree in Civil Engineering from Faculty of Civil Engineering, University of Rijeka in Croatia. She is currently studying as a PhD student in the Institute of Structural Engineering at University of Natural Resources and Life Sciences Vienna, Austria. Her research interests are life cycle assessment and maintenance plans of existing reinforced concrete bridges and durability of protection and repair systems of concrete structures.

Alfred STRAUSS. Prof. Alfred Strauss, born 1968 studied Civil Engineering at the Technical University of Vienna. He received a Full Professor Qualification (Scientific Habilitation) from the University in 2013. His education includes a 5yr Degree in Civil Engineering with honours from the Technical University of Vienna (1997), a PhD in Stochastic-Probabilistic Engineering from University of Natural Resources and Life Sciences (2004). He is an experienced researcher and consultant engineer for advanced structural analysis problems related to bridges, roof systems, and off shore structures. His main research areas include computational analysis of concrete structures, structural reliability, structural optimization, life-cycle engineering and artificial intelligence in structural engineering.

Jose C. MATOS. He is an assistant professor at the Civil Engineer Department of Minho University. He is Chairman of COST Action TU 1406 (www.tu1406.eu). His main research areas include probabilistic-based assessment of existing structures and bridges and structures management systems.

Joao AMADO. Civil Engineering degree and post-graduation from University of Coimbra, MSc degree from NOVA University of Lisbon. Work experience from construction to consultancy with special emphasis in bridge structural inspections, testing, monitoring and infrastructure's management systems. Currently works at Infraestruturas de Portugal, general concessionaire for roads and railways in Portugal, in the field of asset management. Published works in the scope of development, monitoring and systematization of management systems. 